\title{
High statistic analysis of nucleon form factors and charges in lattice QCD
}

\section{Eigo Shintani*}

RIKEN Advanced Institute for Computational Science, Kobe, Hyogo 650-0047, Japan

E-mail: shintani@riken.jp

\section{Hatmut Wittig}

PRISMA Cluster of Excellence, Institut für Kernphysik,

Johannes Gutenberg-Universität Mainz, D-55099 Mainz, Germany

E-mail: hartmut.wittig@uni-mainz.de

\begin{abstract}
We systematically study the effect of excited state contamination into the signal of nucleon axial, (iso-)scalar and tensor charge, from three-point function with various sets of source-sink separation. In order to enhance the statistics to $\mathrm{O}(10,000)$, we use the all-mode-averaging technique using the approximation of observable to reduce the computational cost. Numerical study is performed with the range of source-sink separation $\left(t_{s}\right)$ from $0.8 \mathrm{fm}$ to more than $1.5 \mathrm{fm}$ with cut-off scales, $a=0.063 \mathrm{fm}$, and pion masses, $m_{\pi}=0.277 \mathrm{GeV}$, keeping the volume as $m_{\pi} L>4$ on $N_{f}=2$ Wilson-clover fermion configurations in Mainz-CLS group. In the analysis using $t_{s}>1.5 \mathrm{fm}$, the axial charge approaches to experimental result near physical point.
\end{abstract}

The 33rd International Symposium on Lattice Field Theory

14 -18 July 2015

Kobe International Conference Center, Kobe, Japan

\footnotetext{
* Speaker.
} 


\section{Introduction}

Recent development of the numerical algorithm, software and hardware makes a tremendous progress to be exactly simulating the dynamics of nucleon and nuclei including the dynamical light quarks close to physical pion mass in lattice QCD. In fact, for instance, the lattice computation of hadron spectrum allows us to precisely evaluate its spectrum including not only ground state but also broad channel of resonance state.

On the other hand, aiming for the precise calculation of nucleon structure function in lattice QCD, we confront a dilemma, which is a sort of noise issue when pursuing the high precision of Monte-Carlo simulation removing the contamination of non-ground state (excited state) effect. According to a naive expectation of signal-to-noise ratio of nucleon propagator given its variance as described by induced pion propagator, $\mathrm{S} / \mathrm{N} \sim \sqrt{N} \exp \left[-\left(m_{N}-3 / 2 m_{\pi}\right) t\right]$, in statistics $N$ at temporal extension $t$, its signal becomes exponentially small rather than statistical noise, even approaching to the light quark, while $\mathrm{S} / \mathrm{N}$ is scaled as a function of only square of independent statistics.

This study aims to reduce the statistical noise even in large time-separation by using the recent numerical technique, all-mode-averaging (AMA) $[1,2,3]$, applying to various lattice parameters, cut-off scale, pion masses and lattice volume, and rigorously test the consistency of several analysis suggested by $[4,5,6,7,8]$. Our analysis has been carefully carried out focusing on the dependence of source-sink separation and our result suggests that lattice computation of axial charge at $a=$ $0.063 \mathrm{fm}$ in $3 \mathrm{fm}^{3}$ volume using $m_{\pi}=0.277 \mathrm{GeV}$ (whose gauge ensemble is called as "F7") has significant effect of excited state contamination, and analysis taking into account large source-sink separation allows us to make it close to experimental value. As we present in this proceedings, it also provides quantitative information towards future study of nucleon form factor in the physical point.

\section{High statistics study of excited state contamination in two-point function}

In this section, we study the excited state contamination of the nucleon two-point function. Assuming that the two-point function at $t>0.5 \mathrm{fm}$ is dominated by the ground and first excited state, the two-point function is described as a double exponential function (two-state fitting),

$$
G_{N}(t)=\operatorname{Tr}\left[P^{+}\langle N \bar{N}\rangle(t)\right] \simeq Z_{N} e^{-m_{N} t}+Z_{N^{\prime}} e^{-m_{N^{\prime}} t},
$$

with mass of nucleon ground state $m_{N}$ and its first excited state $m_{N^{\prime}}$, which are free parameters in chi-squared fitting. Since the mass of higher excited state is much larger than ground state, twopoint function will be dominated by two contribution of ground and the first excited state at larger time-separation than $t \simeq 0.5 \mathrm{fm}$ from source location. In order to observe the region of ground state dominance and appearance of the leading excited state contamination, it is convenient to show the effective mass, in which constant-like behavior in ground state dominance at large $t$ appears and curvature due to exponential function of mass difference between ground and excited state is observed in short $t$. In Figure 1, one sees that effective mass has a plateau region starting from $t \simeq 1.0 \mathrm{fm}$. whose value is also in good agreement with result of point-sink and smeared-source two-point function at $t=1.2 \mathrm{fm}$, and therefore we also estimate the ground state of nucleon mass 

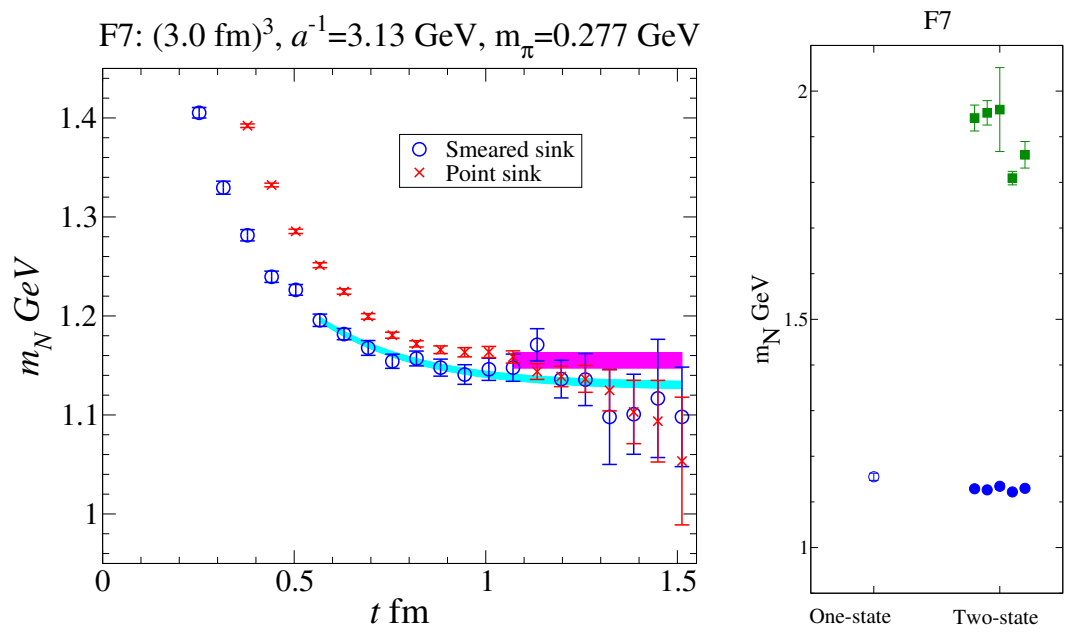

Figure 1: (Left) Effective mass plot of the nucleon on F7 gauge ensemble. The different symbols are the results with different function of sink nucleon operator. The solid band denotes the function of one-state (straight) and two-state (curved) fit including statistical error. (Right) Fitting result with one- and two-state for nucleon two-point function. The several data in two-state ansatz denotes the result in several fitting range

obtained by single exponential function (one-state fitting) using a range of $1.0 \mathrm{fm}<t<1.5 \mathrm{fm}$. On the other hand, below $t=1.0 \mathrm{fm}$, the curvature of excited state contamination clearly appears, with two-state fitting Eq. (2.1), and thus to obtain the first excited state mass we perform chi-squared fitting with a range starting around $t \simeq 0.5 \mathrm{fm}$. One also sees that two-state fitting is well describing the effective mass plot in Figure 1.

In the right panel of Figure 1, we compare the evaluation of the nucleon mass and excited state mass. The value of ground state nucleon mass has slight discrepancy between one- and two-state fit depending on the fitting range, as well as the excited state mass obtained by two-state fitting. This is because the contamination of higher excited state is not clearly distinguished from ground and first excited state by two-state fitting ansatz. In our analysis, $\chi^{2} / \mathrm{dof}$ for correlated fitting of data at each time-slice is less than 5.0 which is not so much large even in two-state fitting. To use the mass difference from ground state and the first excited state in the analysis of three-point function. we choose the central value and statistical error of the first fitting range as the reference point, and then conservatively estimate its systematic error from the maximum difference of central value from the reference point.

\section{High statistics study of excited state contamination in three-point function}

Since the ratios of three- and two-point function, $R_{A}, R_{S}$ and $R_{T}$,

$$
R_{\mathscr{O}}\left(t, t_{s}\right)=\frac{\operatorname{tr}\left[P_{\mathscr{O}}\left\langle N\left(t_{s}\right) \mathscr{O}(t) \bar{N}(0)\right\rangle\right]}{\operatorname{tr}\left[P^{+}\left\langle N\left(t_{s}\right) \bar{N}(0)\right\rangle\right]},
$$

with $P_{A, T}=i \gamma_{5} \gamma_{3}\left(1+\gamma_{4}\right) / 2$ and $P_{S}=\left(1+\gamma_{4}\right) / 2$, have the effect of excited state contamination at finite $t$ and $t_{s}$, we systematically study using variations of $t_{s}$ with high quality data set. Truncating 
the excited state contamination up to the next-to-leading order, the ratio $R_{\mathscr{O}}$ can be described as the two-state contribution, ground and the first excited state,

$$
R_{\mathscr{O}}\left(t, t_{s}\right)=g_{\mathscr{O}}+c_{\mathscr{O}}\left(e^{-\Delta t_{s}}+e^{-\Delta\left(t_{s}-t\right)}\right)+O\left(e^{-\Delta^{\prime}\left(t_{s}-t\right)}\right),
$$

with the target observable $g_{\mathscr{O}}$, mass difference $\Delta$ and unknown coefficient $c_{\mathscr{O}}$. The higher order effect than the first excited state contamination, $\Delta^{\prime}$, is ignored in this analysis, so that it is regarded as a systematic uncertainty. In order to check the consistency, we compare two analysis methods,

- Fitting with the ansatz of Eq. (3.2) in a middle range of $0<t<t_{s}$ (Two-state fitting) [7, 8],

- Summation of $R_{\mathscr{O}}$ over $t_{s}$ and fitting with linear function (Summation method) [10, 4, 5].

where in the first method, we also use the one-state fitting, which is constant fitting assuming the ground state dominance around the middle of $t_{s}$.

\subsection{One-state fitting for axial charge}

We first show the one-state fitting of axial charge. This is ordinal method to extract form factor assuming ignoring excited state contamination, and therefore it is used as a reference point to figure out how large the systematic uncertainty of excited state contamination contains in our fitting range.

Figure 2 plots $R_{A}\left(t, t_{s}\right)$ relying on $t$ with variation of $t_{s}$, and result of constant fitting. One sees that there appears significant contribution of $e^{-\Delta\left(t_{s}-t\right)}$ in Eq.(3.2) and, as a consequence of onestate fitting, it has clear tendency to approach the experimental value from below, which may lead to $e^{-\Delta t_{s}}$ effect due to excited state contamination. Compared to previous study in [5], our analysis makes further clear effect of excited state contamination at $t_{s}$ more than $1.3 \mathrm{fm}$ by the reduction of statistical fluctuation boosted by AMA method. We also notice that in the comparison with recent lattice result in [8], in which they used the $N_{f}=2$ Wilson-clover fermion configurations with various kinds of kappa and volume, our analysis provides the consistent result at same $t_{s}$ with similar accuracy, and furthermore the discrepancy increasing at $t_{s} \geq 1.5 \mathrm{fm}$ may be suggestive that it is necessary to use larger $t_{s}$ than $1.5 \mathrm{fm}$ for avoidance of underestimate the systematic uncertainty of excited state contamination into $g_{A}$ before proceeding to estimate of other systematic uncertainty, for instance, finite size, pion mass dependence and cut-off correction.

\subsection{Two-state fitting for axial charge}

Once turning on the excited state term in a fitting function Eq. (3.2), it allows us to explicitly see the correction of exponent $e^{\Delta t}$ to $g_{A}$. Since the source and sink functions are set up to be same at zero momentum transfer, there appears same contribution of excited state from source and sink location to operator location $t$, and so that taking average over the lattice data folding at middle point of $t_{s}$ is also useful to enhance the statistical accuracy without any additional computation. Figure 3 shows the two-state fitting with folded $R_{A}$. The $\chi^{2}$ fitting is working well, and those values have comparable accuracy with one-state fitting with $t_{s}>1.3 \mathrm{fm}$. One also sees that there is significant correction of the first excited state below $t_{s} \leq 1.2 \mathrm{fm}$, and then axial charge obtained by two-point function becomes stable even when varying $t_{s}$ from $0.8 \mathrm{fm}$ to $1.3 \mathrm{fm}$. We also observe 

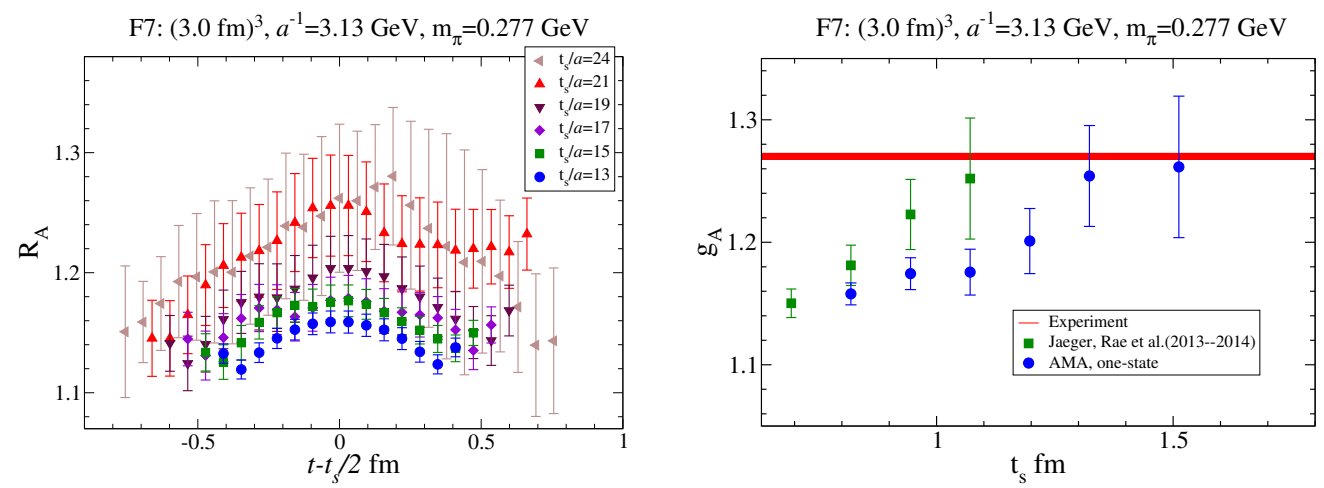

Figure 2: (Left) $R_{A}\left(t, t_{s}\right)$ for nucleon axial charge as a function of $t-t_{s} / 2$ with variation of $t_{s}$ on each gauge ensemble. (Right) $g_{A}$ as a function of $t_{s}$ obtained by constant fitting of $R_{A}\left(t, t_{s}\right)$. For the comparison, green-squared symbols denote the previous results in [5] on the same configurations but without AMA.
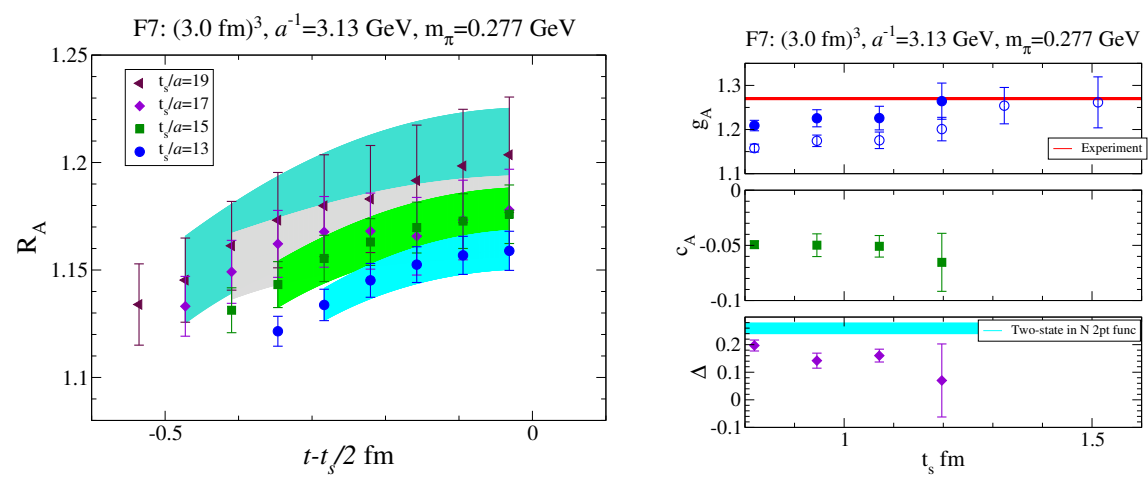

Figure 3: (Left) $R_{A}$ with folding at middle point of $t_{s}$. Different symbols are lattice data with each input $t_{s}$. The solid bound denotes the fitting function with two-state fitting. (Right) The fitting parameter obtained by two-state fitting, $g_{A}, c_{A}$ and $\Delta$. The blue circles are the result with one-state fitting. Red colored bound is experimental result, and cyan colored bound denotes the mass difference obtained by two-state fitting of nucleon two-point function.

that the $\Delta$ evaluated by two-state fitting of $R_{A}$ is similar value with $\Delta$ obtained by two-state fitting with two-point function as shown in Section 2. It turns out that the curvature on the edge of $R_{A}\left(t, t_{s}\right)$ is almost dominated by the mass difference between the first and excited state as well as appearing in two-point function. This provides useful information to carry out the same systematic analysis on the new configurations [9] in the future.

\subsection{Summation method for axial charge}

Taking a summation of $R_{A}\left(t, t_{s}\right)$ over $t_{s}$, it can remove the contribution of $e^{-\Delta\left(t_{s}-t\right)}$ in Eq. (3.2),

$$
R_{\mathscr{O}}^{\mathrm{sum}}\left(t_{s}\right) \equiv \sum_{t=1}^{t_{s}} R_{\mathscr{O}}\left(t, t_{s}\right)=d_{1}+\left(g_{\mathscr{O}}+O\left(e^{-\Delta t_{s}}\right)\right) t_{s}
$$

in which $\Delta$ appears as the mass gap between ground and the first excited state. Compared to twostate fitting, the number of fitting parameter is reduced to two parameters, which are the target 

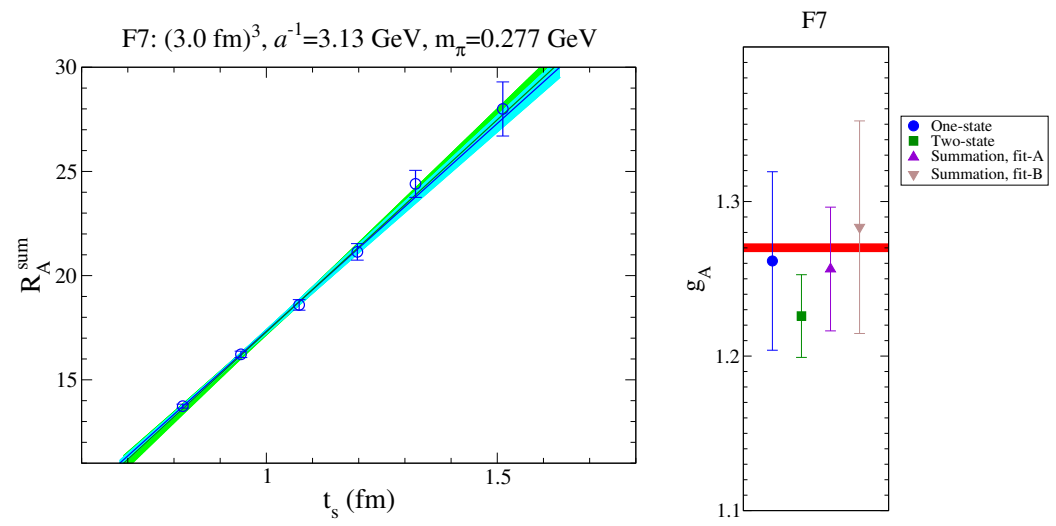

Figure 4: (Left) $R_{A}^{\text {sum }}$ as a function of $t_{s}$ on each gauge ensemble. The solid lines are linear function with a fitting range of all $t_{s}$ points (fit $\mathrm{A}$ ) (blue line denotes central value and band denotes statistical error) and using from the next-to-minimum to maximum $t_{s}$ (fit B) (green line and band). (Right) $g_{A}$ obtained from four methods (one-state, two-state, summation with fit range A, B). The red band shows the experimental result.

observable $g_{\mathscr{O}}$ and the coefficient $d_{1}$ which is merged with excited state contamination. In order for valid estimate of observable $g_{\mathscr{O}}$ with summation method it is required of enough accuracy of three-point function at large $t_{s}$ to ignore $O\left(e^{-\Delta t_{s}}\right)$ in the second term, and perform the linear fitting within large $t_{s}$ region. Figure 4 shows that our data points seem to be a linear dependence for $t_{s}$. Compared to two fitting regions in which one is using all $t_{s}$ points (fit $\mathrm{A}$ ) and the other is starting from $t_{s}>0.9 \mathrm{fm}$ (fit B), there does not see the significant difference between them.

\subsection{Iso-scalar and tensor charge}

Finally we show the study of excited state contamination for iso-scalar $g_{S}$ and tensor charge $g_{T}$, which are extracted from the ratio of three-point function including scalar and tensor operator. Figure 5 shows renormalized $g_{S}$ and $g_{T}$ obtained from the one-state fitting with same fitting range as in $g_{A}$. One sees that, contrasting with $g_{A}$, the lattice result changing $t_{s}$ from $1 \mathrm{fm}$ to $1.5 \mathrm{fm}$ does not significantly rely on $t_{s}$, and so that there is not appearance of excited state contamination. In addition, we do not observe the discrepancy from [8] with similar lattice parameter. It turns out that for $g_{S}$ and $g_{T}$ the excited state contamination is controlled well even in $t_{S} \simeq 1 \mathrm{fm}$ as argued in $[7,8]$.

\section{Summary}

We investigate the excited state contamination effect of nucleon form factor calculation, especially for axial charge, in lattice QCD using high statistics at large source-sink separation more than $t_{s}=1.5 \mathrm{fm}$. By applying the all-mode-averaging (AMA) technique to enhance the statistics without additional computational cost, it enables us to investigate the systematic behavior of axial charge, iso-scalar and tensor charge as relying on the source-sink separation. As a consequence of consistency test of two different analysis, i.e. one- or two-state fitting and summation method, above $t_{s} \simeq 1 \mathrm{fm}$, we see that $g_{A}$ extracted from the ratio of three- and two-point function is suffered from large contribution of excited state contamination, although $g_{S}$ and $g_{T}$ are not. This is feasible 

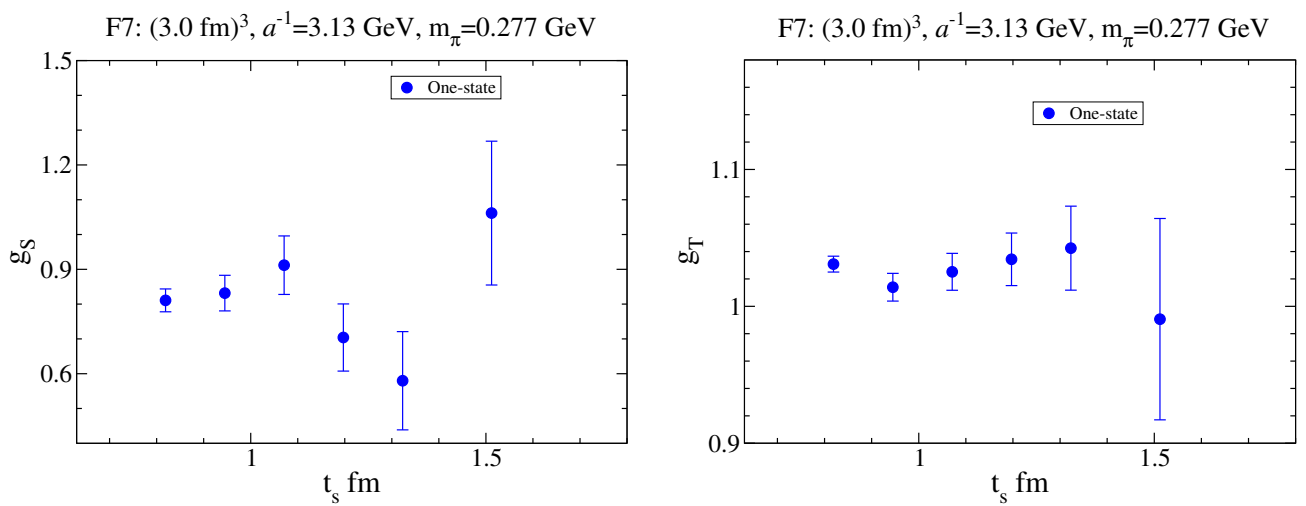

Figure 5: Renormalized iso-scalar charge $g_{S}$ (left) and $g_{T}$ (right) at $2 \mathrm{GeV}$ scale as a function of $t_{s}$ with one-state fitting on each ensemble.

study of determination of physical value of nucleon form factor from lattice QCD, and so that we will perform further study of the chiral behavior of nucleon form factor including not only $g_{A}$ but also iso-vector channel with various lattice parameters [6] in high statistics under way.

Our calculations were performed on the "Wilson" and "Clover" HPC Clusters at the Institute of Nuclear Physics, University of Mainz. We thank Dalibor Djukanovic and Christian Seiwerth for technical support. This work was granted access to the HPC resources of the Gauss Center for Supercomputing at Forschungzentrum Jülich, Germany, made available within the Distributed European Computing Initiative by the PRACE-2IP, receiving funding from the European Community's Seventh Framework Programme (FP7/2007-2013) under grant agreement RI-283493 (project PRA039).

\section{References}

[1] T. Blum, T. Izubuchi and E. Shintani, Phys. Rev. D 88, no. 9, 094503 (2013) [arXiv:1208.4349 [hep-lat]].

[2] T. Blum, T. Izubuchi and E. Shintani, PoS LATTICE 2012, 262 (2012) [arXiv:1212.5542].

[3] E. Shintani, R. Arthur, T. Blum, T. Izubuchi, C. Jung and C. Lehner, Phys. Rev. D 91, no. 11, 114511 (2015) [arXiv:1402.0244 [hep-lat]].

[4] S. Capitani, M. Della Morte, G. von Hippel, B. Jager, A. Juttner, B. Knippschild, H. B. Meyer and H. Wittig, Phys. Rev. D 86, 074502 (2012) [arXiv:1205.0180 [hep-lat]].

[5] B. Jäger et al., PoS LATTICE 2013, 272 (2014) [arXiv:1311.5804 [hep-lat]].

[6] S. Capitani et al., Phys. Rev. D 92, no. 5, 054511 (2015) [arXiv:1504.04628 [hep-lat]].

[7] T. Bhattacharya, S. D. Cohen, R. Gupta, A. Joseph, H. W. Lin and B. Yoon, Phys. Rev. D 89, no. 9, 094502 (2014) [arXiv:1306.5435 [hep-lat]].

[8] G. S. Bali et al., Phys. Rev. D 91, no. 5, 054501 (2015) [arXiv:1412.7336 [hep-lat]].

[9] M. Bruno et al., JHEP 1502, 043 (2015) doi:10.1007/JHEP02(2015)043 [arXiv:1411.3982 [hep-lat]].

[10] S. Gusken, U. Low, K. H. Mutter, R. Sommer, A. Patel and K. Schilling, Phys. Lett. B 227, 266 (1989). 